\title{
A DNA replication-arrest site $R T S 1$ regulates imprinting by determining the direction of replication at mat1 in $S$. pombe
}

\author{
Jacob Z. Dalgaard ${ }^{1-3}$ and Amar J.S. Klar ${ }^{2}$ \\ ${ }^{1}$ Marie Curie Research Institute (MCRI), The Chart, Oxted, Surrey RH8 0TL, UK; ${ }^{2}$ Department of Health and Human \\ Services (DHHS), National Cancer Institute, Division of Basic Sciences, Gene Regulation and Chromosome Biology \\ Laboratory, Frederick Cancer Research and Development Center, Frederick, Maryland 21702-1201, USA
}

\begin{abstract}
Mating-type switching in Schizosaccharomyces pombe involves a strand-specific, alkali-labile imprint at the mat1 (mating-type) locus. The imprint is synthesized during replication in a swi1, swi3, and polymerase $\alpha$ (swi7) dependent manner and is dependent on mat1 being replicated in a specific direction. Here we show that the direction of replication at mat1 is controlled by a cis-acting polar terminator of replication (RTS1). Two-dimensional gel analysis of replication intermediates reveals that RTS1 only terminates replication forks moving in the centromere-distal direction. A genetic analysis shows that RTS1 optimizes the imprinting process. Transposing the RTS1 element to the distal side of mat1 abolishes imprinting of the native mat1 allele but restores imprinting of an otherwise unimprinted inverted mat1 allele. These data provide conclusive evidence for the "direction of replication model" that explains the asymmetrical switching pattern of $S$. pombe, and identify a DNA replication-arrest element implicated in a developmental process. Such elements could play a more general role during development and differentiation in higher eukaryotes by regulating the direction of DNA replication at key loci.
\end{abstract}

[Key Words: Differentiation; imprinting; mating-type switching; Schizosaccharomyces pombe; termination of DNA replication]

Received February 14, 2001; revised version accepted June 13, 2001.

The mating type of Schizosaccharomyces pombe is determined by the alternate alleles of the mat1 locus, which are specific to the $\mathrm{P}$ (plus) or $\mathrm{M}$ (minus) cell type (for review, see Egel 1989; Dalgaard and Klar 2001). Mating-type switching occurs when the mat1 allele is replaced with the opposite mating-type allele by a recombination event. This event copies the incoming information from one of the two transcriptionally silent donor cassettes, mat2P or mat $3 M$, located centromere-distal to mat1 (Fig. 1A, upper; Beach 1983; Beach and Klar 1984). Pedigree studies have shown an interesting asymmetrical pattern of mating-type switching (Fig. 1B, bold letters; Miyata and Miyata 1981; Egel and Eie 1987; Klar 1987, 1990). For each mating type there are switchable and unswitchable cells. When an unswitchable cell divides, two cells are formed of the parental mating type of which only one is switchable. When a switchable cell divides, it gives rise to a switched cell of opposite mating type, plus a switchable cell of the parental mating type. As a result, starting with an unswitchable cell, after two

${ }^{3}$ Corresponding author.

E-MAIL j.dalgaard@mcri.ac.uk; FAX +44-1883-714-375.

Article and publication are at http://www.genesdev.org/cgi/doi/10.1101/ gad.200801. divisions, only one of four granddaughter cells switches mating type, whereas two other granddaughter cells are switchable (Fig. 1B, insert). This pattern is due to a strand-specific modification (imprint) that marks the DNA at the mat1 locus of switchable cells (Egel and Eie 1987; Klar 1987, 1990). The imprint has been shown to be either a strand-specific nick (Arcangioli 1998) or an alkali-sensitive modification of the DNA strand (Dalgaard and Klar 1999). Imprinting is dependent on mat1 cis-acting sequences (Arcangioli and Klar 1991; Styrkarsdottir et al. 1993) and on several trans-acting factors including swi1, swi3, and swi7 (Egel et al. 1984). Of these trans-acting factors the swi7 gene encodes DNA polymerase $\alpha$, which is primarily involved in lagging-strand synthesis (Singh and Klar 1993).

A model named the direction of replication model has been proposed to explain the asymmetrical pattern of mating-type switching (Dalgaard and Klar 1999). This model suggests that the DNA replication machinery is involved in imprinting as well as in initiation of switching (Fig. 1B, line drawing; Dalgaard and Klar 1999). The model proposes that the imprint is synthesized, or formed, by the lagging-strand replication complex but only when this complex synthesizes the upper strand at 
A

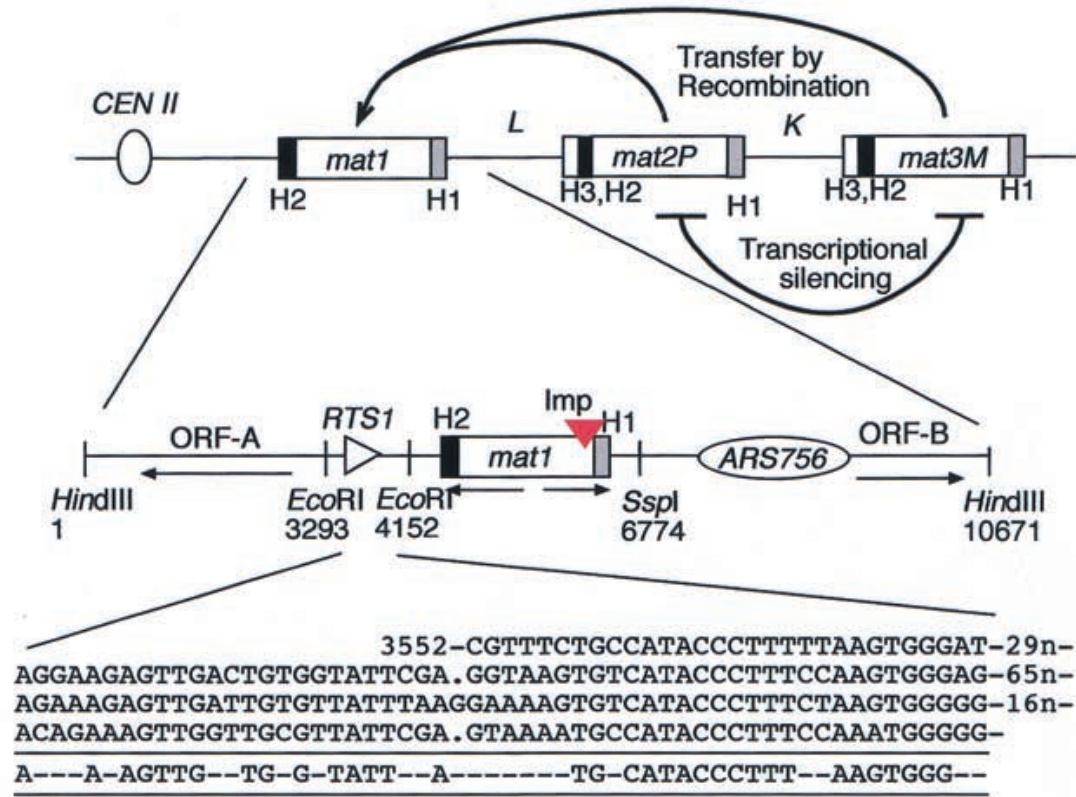

B

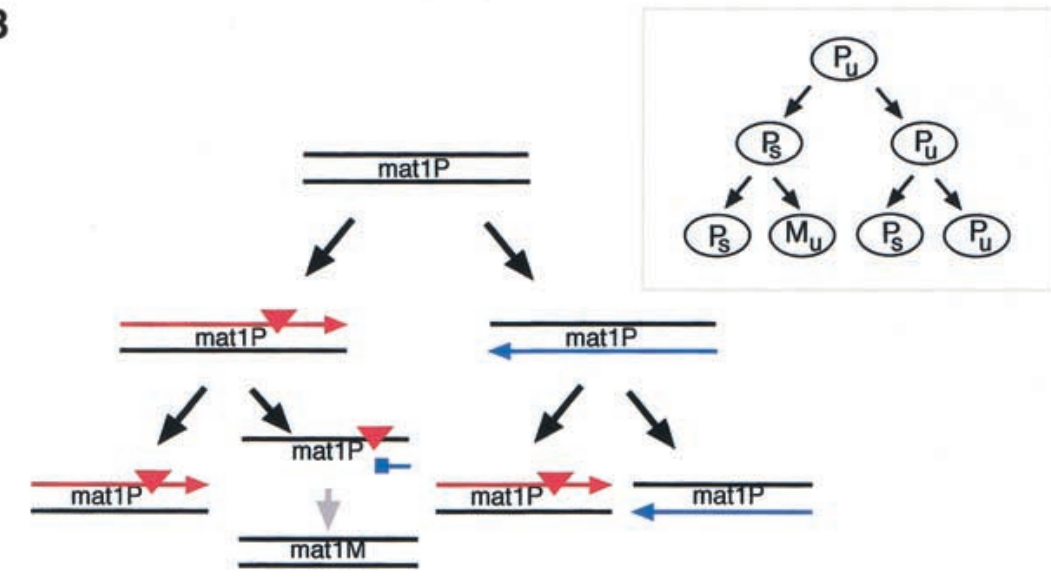

Figure 1. The mat1 region and the switching pattern of $S$. pombe. $(A)$ Outline of the mating-type region. The arrows illustrate substitution of mat1. $\mathrm{H} 1, \mathrm{H} 2$, and $\mathrm{H} 3$ are homology boxes, (CEN) Centromere. Below, a graphic outline of the sequence of the 10.7-kb HindIII fragment containing mat1P. The positions of the identified ORFs (horizontal arrows), the repeats (open triangle), and ARS756 are indicated. An alignment of the RTS1 repeats is given below. The numbers of nucleotides separating the repeats are shown on the right. The consensus of the repeat sequences is shown below the alignment. (Imp) Imprint (red triangle). (B) The direction of replication model (Dalgaard and Klar 1999) and pedigree of switching. Black arrows indicate the inheritance of a particular DNA chain by the specific daughter cell. The mat1 cassette is replicated by distally located origins and only the lagging strand (red arrow) is imprinted during its synthesis. The cell inheriting the imprinted chromosome becomes switchable. Leading-strand replication (blue arrow) in a switchable cell is postulated to result in a transient break due to the inherited modification in the template DNA. The transient break initiates recombination resulting in the mating-type switch (vertical gray arrow). The insert displays the corresponding pedigree of switching starting with a P cell. Subscripts " $\mathrm{s}$ " and " $\mathrm{u}$ " designate switchable and unswitchable cells, respectively. mat1. As a result only one of the two sister chromatids synthesized during replication becomes imprinted. The cell that inherits the imprinted chromosome becomes switchable, whereas the sister cell remains unswitchable. Mating-type switching is proposed to initiate during the next round of replication when the leading-strand replication complex encounters the imprint. A break is formed in only one of the two sister chromatids synthesized and this break induces a recombination event that leads to mating-type switching. Consistent with the direction of the replication model, mat1 is replicated unidirectionally from centromere-distal origins, and imprinting is sensitive to changes in the direction of replication at mat1 (Dalgaard and Klar 1999). Furthermore, a replication-pause site, named MPS1, has been identified at the site of imprinting (Dalgaard and Klar 1999, 2000). Pausing at MPS1 depends on Swil and Swi3 functions, as well as the direction of replication, suggesting that pausing of the replication fork is an essential part of the imprinting process. The latter observation also provides a mechanistic explanation for why the imprinting process is sensitive to changes in the direction of replication (Dalgaard and Klar 2000). Interestingly, a replication barrier, named RTS1, which is also dependent on the transacting factors Swi1 and Swi3 for function, is located centromere-proximal to mat1 (Dalgaard and Klar 2000).

This work shows that the RTS1 barrier is a polar terminator of replication that optimizes imprinting by controlling the direction of replication at mat1. Optimization of imprinting occurs by termination of replication forks moving in the distal direction, thereby preventing these from replicating mat1 and interfering with imprinting. Furthermore, using the RTS1 element, genetic rearrangements in the mat 1 region provide additional conclusive evidence for the direction of replication model.

\section{Results}

Analysis of mat 1 replication intermediates identifies a polar replication-termination site

To identify cis-acting elements that may be involved in 
establishing the direction of replication, the $10.7-\mathrm{kb}$ HindIII mat1 fragment was sequenced and analyzed (Fig. 1A, middle). The analysis showed that a mat1-distal region between nucleotides 6980-9678 contains a autonomous replicating sequence identified previously (ARS756; Maundrell et al. 1988). Analysis of the mat1 proximal sequence identified a cluster of partially conserved direct repeated sequence motifs located $\sim 0.5 \mathrm{~kb}$ left of the mat1 locus (Fig. 1A, lower panel). The cluster contains three full-length copies plus one truncated copy. The repeat sequence is $\sim 60$ bp long with two subdomains of higher sequence conservation. Computer searches with the 60-bp repeat sequence did not detect its presence at other places in the $S$. pombe genome.

To determine whether the potential origin or the repeats control the direction of replication at mat1, replication intermediates were analyzed using the two-dimensional (2D) gel technique (Brewer and Fangman 1987; Fig. 2). The method separates restricted replication intermediates on 2D agarose gels for subsequent Southern analysis. The analysis was performed with a strain deleted for the donor loci mat2P and mat3M (Klar and Miglio 1986) and containing an additional cis-acting deletion mat1-MsmtO (Styrkarsdottir et al. 1993) that abolished mat1 imprinting. This strain (JZ108) was used because switching intermediates of standard strains (J. Dalgaard, unpubl.), as well as hybridization signals from the donor-loci complicated the interpretation of results. Analysis of the mat1 distal region verified that ARS756 acted as an origin of DNA replication (bubble-arc; Fig. 2,

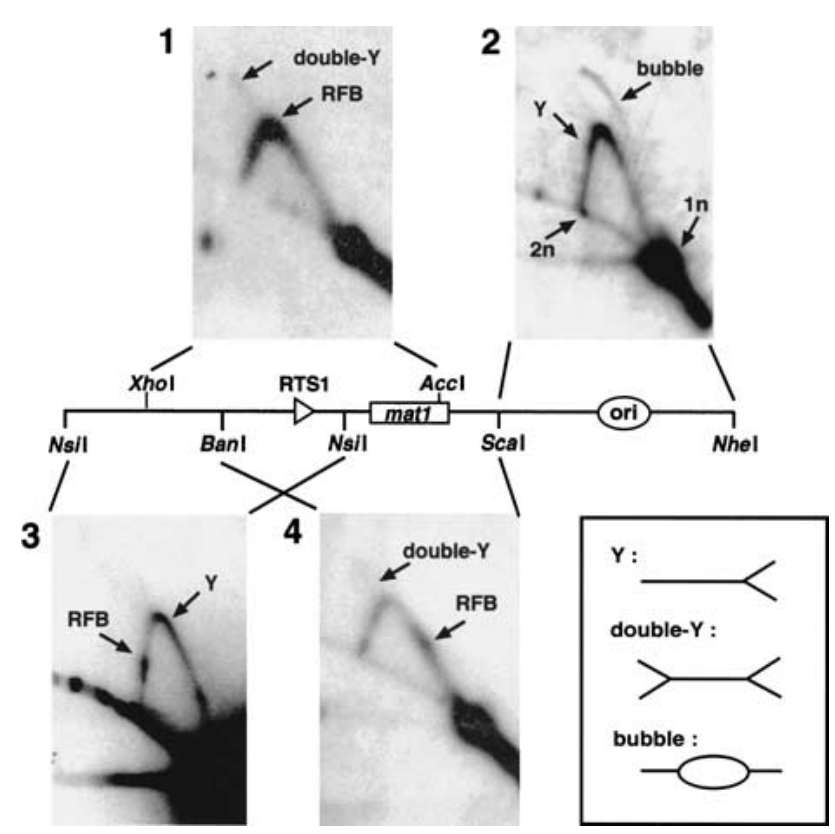

Figure 2. Characterization of replication intermediates of the mat1 region. Standard two-dimensional gel analysis of indicated fragments spanning the mat1 region are shown. Restriction sites used in the analysis are given. (RFB) Replication fork barrier; ( $1 \mathrm{n})$ linear DNA; $(2 \mathrm{n})$ almost fully replicated Y intermediate; (ori) origin. The insert to the right of panel 4 displays a graphic representation of the replication intermediates. panel 2) in a subset $(\sim 5 \%-10 \%)$ of the cell population. However, this low level of initiation of replication is insufficient to confer the predominantly unidirectional mat1 replication (Dalgaard and Klar 1999).

Next, another possibility was examined, whereby the mat1 proximal repeat sequences act as a replication fork barrier (RFB), similar to the Ter elements in prokaryotes (Wake and King 1997), arresting or pausing replication forks initiated by origins proximal to mat1. Such a barrier could optimize imprinting by preventing these replication forks from replicating the mat1 locus. A 2D gel analysis of the mat1 proximal sequences did indeed detect a weak RFB and a double Y-arc characteristic of replication termination (Brewer and Fangman 1988; Fig. 2, panel 1). By analysis of overlapping fragments the RFB was mapped to the repeat region (Fig. 2, panels 1, 3, and 4). Furthermore, the change in position of the RFB on the different arcs indicated that only the forks moving in the distal direction were paused or terminated. However, the low intensity of the RFB and of the double-Y formed by termination at the barrier suggests that only a fraction of the forks responsible for replicating this region terminated at this position.

To further test whether the RFB only terminate or pause replication forks moving in one direction, the direction of fork movements in this region was determined. This technique separates intermediates generated by replication forks moving in one direction from those generated by forks moving in the opposite direction. This technique relies on a second enzymatic digest that is performed after the replication intermediates have been separated in the first dimension (Brewer and Fangman 1988). The second enzymatic digest allows separation of replication intermediates formed by replication fork moving in opposite direction (Fig. 3B). Comparison of the intensity of the two arcs (Fig. 3A) showed that the majority of the replication forks move in a proximal direction, and these are not paused or terminated at the repeats. However, the smaller fraction of forks moving in the distal direction displays a very strong barrier signal at the position corresponding to the repeats. The observation that the arc observed in Figure 3 do not return to the 2n-position, corresponding to a fully replicated Y-intermediate, as well as the observation of a double-Y arc in the previous experiments (Fig. 2, panel $1)$, show that termination, in contrast to pausing, is occurring. This genetic element was therefore named replication termination sequence 1 (RTS1).

To further test the ability of the RTS1 element to act as a polar barrier for replication forks, two plasmids were constructed where the RTS1 element was inserted, in opposite orientations, next to the origin of replication (pBZ141 and pBZ142). Two-dimensional gel analysis of replication intermediates verified that the plasmid-borne RTS1 element only acted as a replication barrier when it was replicated in what corresponds to the mat1-distal direction (Fig. 4, orientation 1). Although a very strong barrier signal was observed, pausing and termination occur as both standard-Y and double-Y arcs were observed. The only partial termination observed could be because 


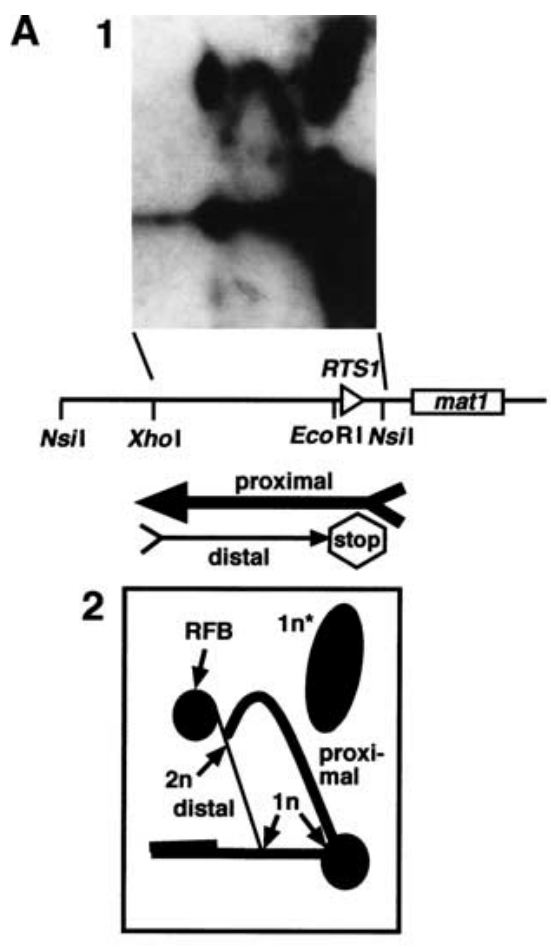

B 1st dimension separation

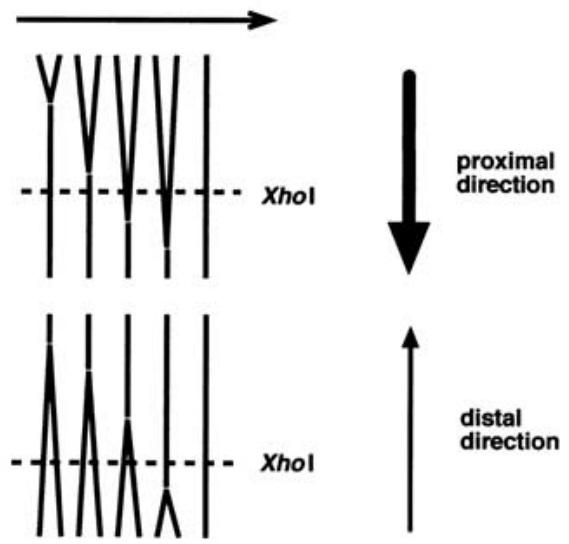

Figure 3. Direction of replication at the mat1-proximal region. (A) Panel 1 shows an autoradiograph of a two-dimensional gel. The blot was probed with the indicated XhoI-EcoRI fragment. The DNA was digested with NsiI before separation in the first dimension, and subsequently the agarose-embedded DNA was digested with XhoI before separation in the second dimension. A schematic drawing of the replication forks passing in one or the other direction is shown below the autoradiograph. The thickness of the lines roughly indicates the relative intensity of the observed arcs. Panel 2 shows the interpretation and schematic drawing of the autoradiograph. $\left(1 n^{\star}\right)$ Linear fragments undigested by the second enzymatic digest used for this method. Remaining symbols are explained in Fig. 1 legend. (B) Diagram explaining the method used. Intermediates are drawn as they are separated by electrophoresis in the first dimension. Intermediates formed by replication forks moving in the proximal direction are drawn above those formed by replication forks moving in the distal direction. The second digest (XhoI) changes the shape of the intermediates, thereby allowing separation of the two types in the second dimension. The direction of the fork movements is given to the left as arrows. the plasmid used in this study is a multicopy plasmid, potentially depleting trans-acting factors involved in termination of replication. RTS1 did not act as a RFB in the plasmid assay when it was replicated in a mat1-distal direction (Fig. 4, orientation 2).

\section{Genetic analysis of cis-acting elements demonstrates the importance of RTS1 for imprinting}

A deletion analysis was conducted to assess the importance of RTS1 and ARS756 for imprinting and switching (Fig. 5). Both the level of imprinting, measured as the amount of double-stranded break (DSB) at mat1 (by Southern analysis; Beach 1983), and the efficiency of switching to the opposite allele (by iodine staining of individual colonies) were analyzed. The amount of DSB is the most convenient and direct measure of the level of imprinting as the imprint is converted into a break during DNA preparation (Beach 1983; Arcangioli 1998; Dalgaard and Klar 1999). Iodine exposure stains a starch-like compound that is synthesized in spores but not in vegetative cells; colonies that switch efficiently stain black due to mating and sporulation, whereas nonswitching colonies stain yellow (Bresch et al. 1968). As expected, deletion of the origin had no significant effect (Fig. 5, JZ349) as it only initiates replication at low fre-

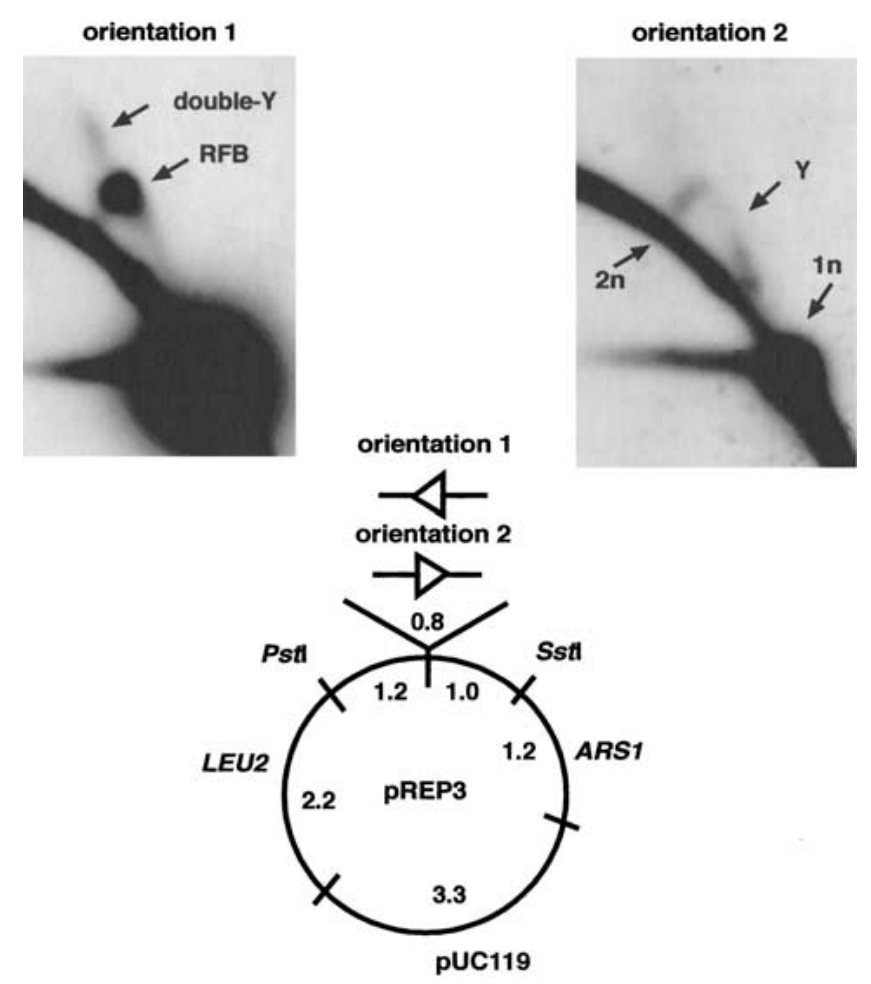

Figure 4. The RTS1 element acts as a polar barrier in a plasmid assay. The 0.8-kb EcoRI fragment was amplified using PCR, introducing BamHI sites at the ends, and cloned into the pREP3 plasmid (Maundrell 1993) in both orientations. The plasmids are outlined below the panels. The sizes of the different plasmid regions are given. The PstI, Sst I fragment indicated in the linedrawing were analyzed for each plasmid by $2 \mathrm{D}$ gel analysis. Blots were probed with the 0.8-kb EcoRI fragment. 
A
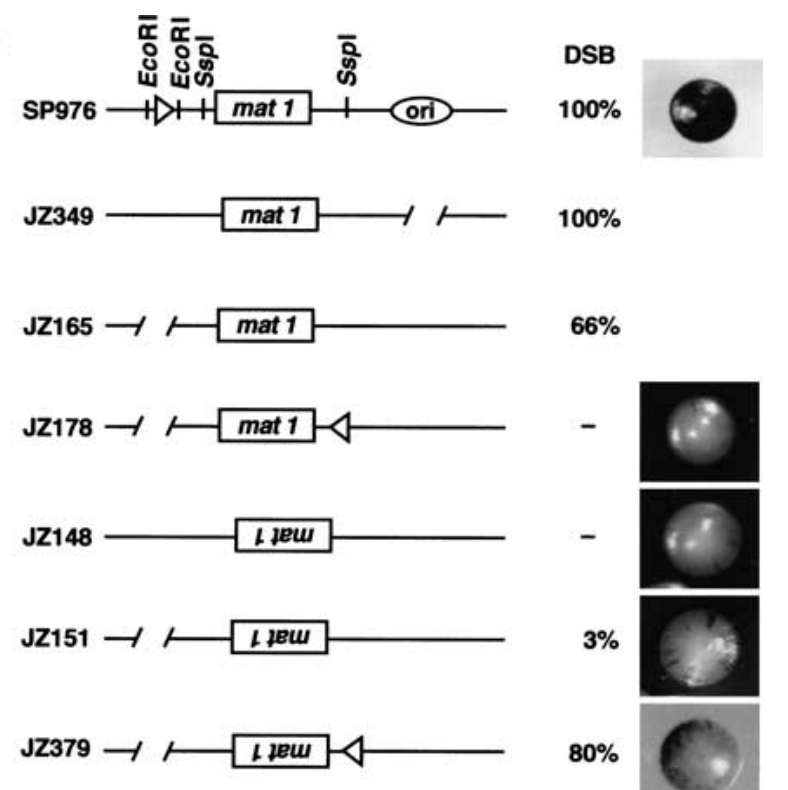

$100 \%$

$66 \%$

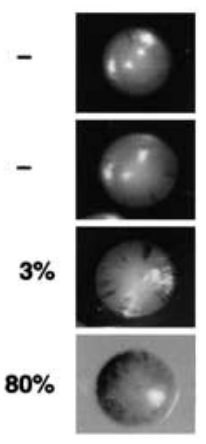

B

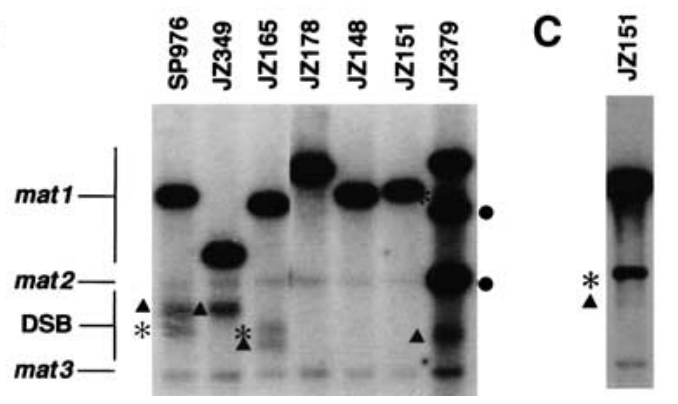

Figure 5. Genetic analysis of the cis-acting elements. $(A)$ Effect of rearrangements on switching. Strain names and schematic drawings of the genetic rearrangements are shown. Strains were constructed using standard cloning and transformation methods (Moreno et al. 1991) from the wild-type strain SP976 (h90, ura4-D18, leu1-32, ade6-M210). The amount of the DSB was determined as described earlier (Dalgaard and Klar 1999) and is presented as the percentage of the value observed for the parental wild-type strain (SP976). For most strains, a colony stained with iodine is also shown. $(B)$ Effect of rearrangements on the DSB level at the mat1 locus. Southern analysis of HindIII-digested chromosomal DNA was performed using the radiolabeled 10.6-kb HindIII mat1P-containing fragment as the probe. mat $2 P$ - and mat $3 M$-containing fragments are visible due to homology with the probe. The proximal and distal fragments, generated by the DSB, are indicated by a triangle and an asterisk, respectively. Closed circles mark signals due to the inversion of the mat1-mat3 regions for strain JZ379. (C) Longer exposure of a lane containing DNA from strain JZ151.

quency. More important, deletion of RTS1 caused a measurable reduction to $\sim 66 \% \pm 15 \%$ of wild-type levels in of the DSB, measured by four independent experiments (JZ165). Pedigree analysis detected also reduction in switching frequency (85\% of the wild-type level). Combining the RTS1 deletion with the origin deletion did not further reduce the DSB level (data not shown). To test whether the RTS1 and ARS756 elements are involved in controlling the replication pattern at mat1, a fork-direction analysis was performed with cells deleted for both elements. First, the swi3 mutation was introduced in the strain JZ349 (JZ375). Swi3 is both involved in termination of replication and imprinting and the mutation as such prevents generation of switching intermediates that otherwise could interfere with the $2 \mathrm{D}$ gel analysis. The 2D gel analysis showed that in the absence of the RTS1 element and the ARS756 origin, mat1 was replicated equivalently in both directions (Fig. 6).

One prediction of the direction of replication model is that if the inverted RTS1 element is inserted distal to mat1 inhibition of imprinting should take place. This effect is expected because mat1 would be replicated in the wrong direction for imprinting. To test the prediction genetically, the EcoRI fragment $(\sim 800 \mathrm{bp})$ containing RTS1 was introduced in an inverted orientation at the mat1 distal SspI site using the Saccharomyces cerevisiae LEU2 gene as a selectable marker (Fig. 5, JZ178). This was done in strain JZ165 that carries a deletion of the endogenous RTS1 element to allow for the passage of forks moving in the distal direction. The RTS1 insertion almost completely abolished switching $(0.3 \%$ sporulation relative to a wild-type control strain) and imprinting (Fig. 5A,B). The observed effect was not due to the LEU2 gene, as insertion of the LEU2 gene by itself did not reduce imprinting and switching (data not shown). Furthermore, a deletion of the LEU2 gene did not restore switching (JZ183; data not shown).

To test whether the effect of this transposed RTS1 element on imprinting was due to a change in the pattern of replication at mat1 a $2 \mathrm{D}$ gel analysis of replication

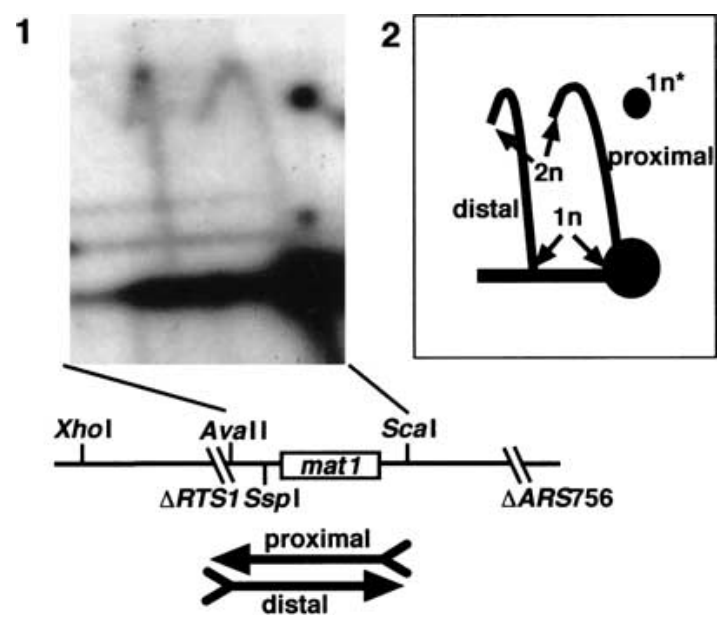

Figure 6. A deletion of RTS1 and distal origin allows bidirectional mat1 replication. (Panel 1) 2D gel analysis of the AvaII$S c a I$ region. The DNA was digested with XhoI and ScaI before separation in the first dimension, followed by digestion with Avall before separation in the second dimension. The blot was probed with the 171-bp AvaII-SspI fragment. A schematic drawing of the region is shown below. The thickness of the lines indicates the relative proportion of the forks moving in indicated directions. (Panel 2) A schematic drawing of the autoradiograph. Symbols are explained in Figure 1 legend. 
intermediates was performed. Analysis of the fragment that contained the introduced RTS1 element detected a remarkably strong RFB (Fig. 7, panel 2). Importantly, because the arc did not return to the $2 \mathrm{n}$ position, corresponding to a fully replicated Y-intermediate (Brewer and Fangman 1987), clearly termination, in contrast to pausing, was occurring. It should be noted that the observed arc and termination signals resemble those observed by Santamaria et al. (2000), where replication forks entering a fragment from both sides simultaneously arrest at a centrally located arrest site. As expected, an analysis of the mat1 proximal region where the RTS1 element had been deleted showed that termination of replication was no longer occurring (Fig. 7A, panel 1). However, two unusual signals were observed (Fig. 7, * intermediates). Analysis of overlapping frag-
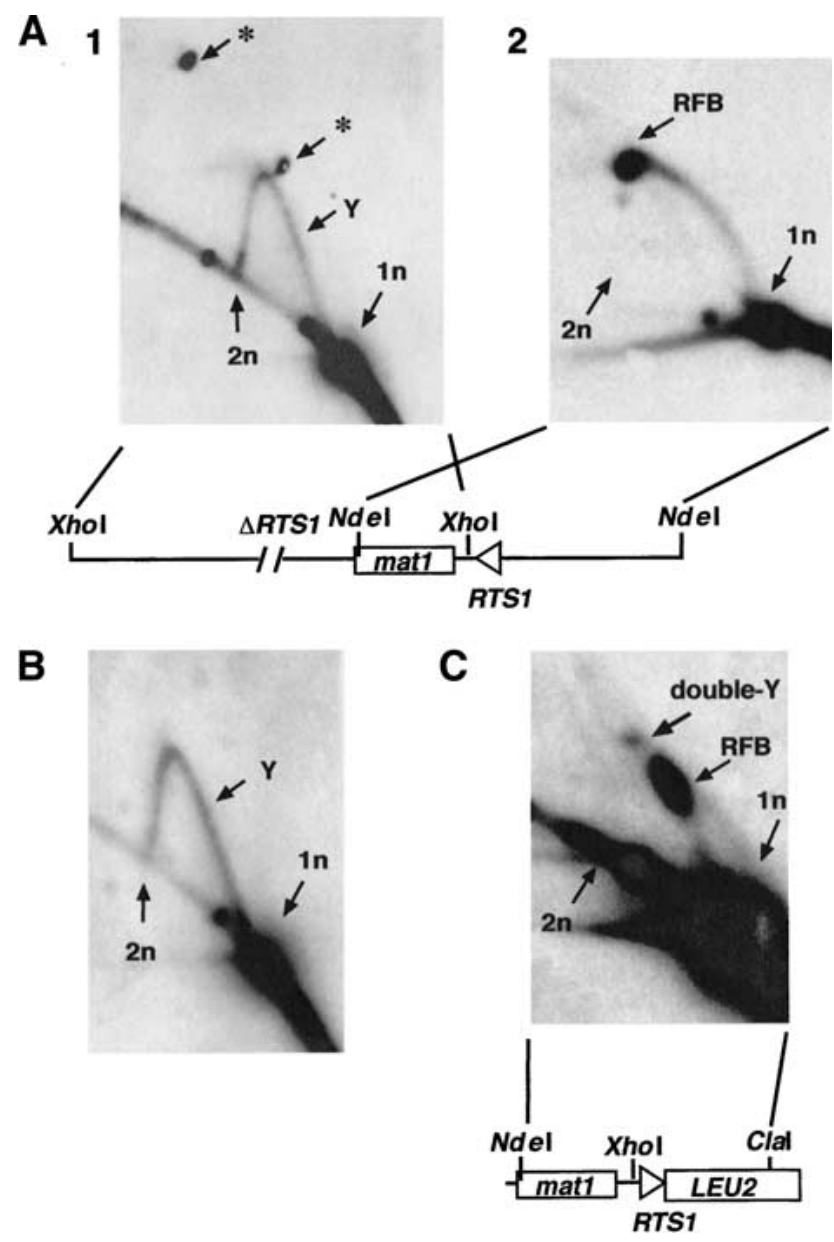

Figure 7. Transposed RTS1 element causes a strong replication block. (A) Standard 2D gel analysis of the mat1 region in strain JZ183 where the inverted RTS1 element is inserted distal to mat1. Enzymes used for the analysis are given in line drawing. The unusual signals are indicated with asterisks. $(B)$ Standard 2D gel analysis of the XhoI fragment (shown above) of strain JZ377 deleted for the donor loci is presented. $(C)$ Standard 2D gel analysis of the mat1 region in strain JZ177 where the RTS1 element is inserted distal to mat1 in the direct orientation. Symbols are explained in Figure 1 legend. ments showed that these signals were only observed when the $0.5-\mathrm{kb}$ region proximal to mat1 was present (data not shown). Furthermore, analysis of a strain deleted for mat $2 P$ and mat $3 M$ donor loci showed that these signals were dependent on the presence of donor cassettes (Fig. 7B, JZ377). However, a more detailed study is needed to address whether a physical interaction between the mat1 cassette and the donor cassettes is taking place in this region to produce these intermediates.

Interestingly, a similar effect on imprinting was observed when the RTS1 element in the direct orientation was inserted on the distal side of mat1 in a strain deleted for the RTS1 element (strain JZ177). The 2D gel analysis showed that termination of replication was occurring at the inserted element (Fig. 7C). This was surprising, as RTS1 at the native position and in the plasmid assay behaves as if it is a polar terminator of replication, and therefore, an effect was only expected when it was inserted in the inverted orientation. This gain of function at the new position might be due to the close proximity of the mat1 pause site MPS1 (see Discussion).

Experiments published earlier have shown that inversion of mat 1 inhibits imprinting, presumably by changing the DNA strand at mat1, which is replicated by the lagging-strand replication complex (Dalgaard and Klar 1999). Another important prediction of the direction of replication model is that restoration of switching should occur when these two rearrangements, which individually abolish imprinting, are combined. First, the indigenous RTS1 was substituted with the ura4 gene in cells containing the inverted mat1 allele (strain JZ148). As expected, a marginal increase in sporulation $(0.3 \%-3 \%)$ and imprinting was observed (Fig. 5A,B, JZ151). This is presumably because of slightly increased replication of mat1 in the distal direction. RTS1 was subsequently introduced at a position distal to the inverted mat1 (JZ379). This introduction led to a remarkable $80 \%$ restoration of imprinting (measured as the DSB level) with only a partial but significant restoration of switching (15\% sporulation relative to the wild type; Fig. 5A,B). The partial restoration of switching was most likely caused by a reduced interaction of the inverted mat1 with the donor loci. It should be noted that the quantification of the imprint was somewhat complicated because of the appearance of a subpopulation of cells carrying an inversion of the entire mat1-mat3M region (Fig. 5B). This rearrangement is formed when a crossover between the inverted mat 1 and mat3 occurred during switching.

\section{Discussion}

This study has characterized cis-acting genetic elements controlling the direction of replication at mat1. The results highlight the involvement of the DNA replication machinery in the developmental program of the lower eukaryote $S$. pombe. The elements include an origin of replication ARS756, located distal to mat1, and the polar terminator of replication, RTS1, located proximal to mat1. The results show that although neither of the 
RTS1 nor ARS756 elements are essential for mat1 imprinting, the RTS1 is especially important in optimizing the switching efficiency by ensuring that the mat1 locus is predominately replicated in the correct orientation for imprinting. The biological function of these cis-acting elements in regulating the direction of replication at the mat1 locus was analyzed using deletions as well as genetic rearrangements. Deletion of the RTS1 element led to a significant reduction in imprinting. However, the most important observation was that transposing the RTS1 element from the proximal to the distal side of mat1 abolished imprinting of a native mat1 allele, and restored switching of an inverted allele. These observations satisfy unique predictions of, and add strong support to, the direction of the replication model, which explains the asymmetrical switching pattern of $S$. pombe, but also explain the molecular mechanism underlying the unidirectional replication at mat1.

Several more detailed observations relating to $S$. pombe mating-type switching can be made from the presented data. First, the presented data exclude the possibility that specialized origins are involved in setting up special replication forks for mat1 imprinting. The observation, that imprinting of an inverted mat1 allele can be restored by transposing the RTS1 element to the proximal side, shows that imprinting at mat1 is not dependent on the initiation of replication from a specific origin located distal to mat1. This result is complemented by earlier experiments where two different origins inserted at the proximal side were able to partly restore switching of the inverted-mat1 allele (Dalgaard and Klar 1999). Therefore, the trans-acting factors involved in imprinting are either an intricate part of the general DNA replication machinery or directly interact with the mat1 locus.

Second, the discovery of the RTS1 element explains earlier results where a significant level of imprinting was observed at a mat 1 cassette inserted in either orientation at the XhoI site located $\sim 2 \mathrm{~kb}$ proximal to mat1 (Klar 1987). The analysis of the proximal mat1 region presented here suggests that replication forks are moving in both directions at this position in the genome. However, a more detailed study is necessary to quantify whether the amount of imprinting correlates with the relative fork movements in those strains. It should be noted that mat1 inverted at the indigenous location fails to imprint/switch (this study), whereas a mat1 inversion (plus the $S$. cerevisiae LEU2 gene sequences) placed $\sim 4.7 \mathrm{kbp}$ to the centromere side imprints fairly well as it switches at a rate of at least 34\% (Klar 1987, 1990). Drastically different behavior of the two inversions is explained by the presence of a polar replication terminator RTS1 residing between the inverted cassettes. In addition, the intervening region is inverted in $~ 50 \%$ of cells, possibly due to intrachromosomal recombinational interaction between mat1 genes in the duplication (A. Klar, unpubl.). Inversion of the RTS1 polar replication terminator will further promote imprinting of the inverted cassette, but the indigenous RTS1 element should reduce imprinting of the directly oriented duplicated cassette. The re- sults (Klar 1987, 1990) are in accordance with these expectations.

Third, during the mating-type switching a process ensures that the mat 1 locus is replaced by separate matingtype information (Thon and Klar 1993). This process is called directionality. Interestingly, two unusual signals were observed by the $2 \mathrm{D}$ gel analysis of the mat 1 proximal region of the strain where the RTS1 element was transposed to the distal side of mat1. The presence of these signals is not fully understood, but suggests that in these strain replication intermediates might resemble a switching intermediate that is temporarily maintained in the cell. The observation that the presence of the signals is dependent on the donor loci suggests that a direct DNA interaction between mat1 proximal sequences and the donor loci is occurring. One interesting possibility is that such an interaction might be important for setting up directionality of switching in $S$. pombe.

S. pombe mating-type switching is the first system where the direction of replication is shown to be important for cellular differentiation (Dalgaard and Klar 1999, 2000). This discovery raises the possibility that the DNA replication machinery or the DNA replication process may play an important role during cellular differentiation and development in higher eukaryotes. One mechanism by which such processes could be regulated is by controlling the direction of replication of specific loci. This could be done by regulating initiation of replication at specific origins or by blocking replication at arrest sites. This work shows that mating-type switching in $S$. pombe relies on a replication arrest site for optimization of imprinting. To our knowledge, this is the first report where a DNA replication-arrest element is implicated in a developmental process. However, such arrest sites could be of more general importance in higher eukaryotes. For example, arrest sites may act by regulating recombination or by promoting differential chromatin assembly of sister chromatids at key developmental loci during replication (Seidman et al. 1979; Shinahara and Stillman 1999). In support of this possibility, differences in the direction of replication have been observed between expressed and unexpressed genes in human cell lines (Trempe et al. 1988; Leffak and James 1989). Whether replication arrest sites are involved in regulation at these genetic loci remains to be explored.

Other DNA replication terminators have been described in eukaryotes but they have been found in arrays of repeated genes: in the rDNA repeats of $S$. cerevisiae (Brewer and Fangman 1987; Linskens and Huberman 1988), S. pombe (Sanchez et al. 1998), plants (Van't Hof and Lamm 1991), Xenopus (Wiesendanger et al. 1994), and human cells (Little et al. 1993), as well at the dihydrofolate reductase locus in Chinese hamster cells (Handeli et al. 1989). In the case of S. cerevisiae, the fob1 replication termination site is involved in regulating the number of repeats present in the rDNA array (Kobayashi et al. 2001). It is not understood how termination of replication is achieved at the RTS1 element. Earlier experiments have shown that the swi1 and swi3 gene products are required for RTS1 function. These genes are also in- 
volved in pausing the replication fork at the MPS1 site necessary for imprinting (Dalgaard and Klar 2000). Only the swil gene has been sequenced and encodes a protein that is homologs to the tim1 gene family responsible for circadian clock formation in eukaryotes. Furthermore, a genetic screen has identified a swil mutant that affects the termination at the RTS1 element without interfering with pausing and imprinting at MPS1 (Dalgaard and Klar 2000). Therefore, one possibility is that Swil and Swi3 pause the replication fork at both these sites, but at the RTS1 site additional trans-acting factors are recruited to mediate termination of replication. The RTS1 element behaves as a polar barrier for replication at its native position and in the plasmid assay. Interestingly, both termination of replication and inhibition of imprinting were observed when RTS1 was inserted in the direct orientations at the distal side at mat1. This is surprising as most replication forks move in the proximal direction at this site and therefore, should pass unhindered by the inserted RTS1 element for subsequent pausing and imprinting at MPS1. However, one possible explanation is that Swil- and Swi3-dependent MPS1 pausing in this strain allows the recruitment of the trans-acting factors involved in termination by the transposed RTS1 elements, thereby causing termination of replication instead of imprinting at MPS1. If this is indeed the case, it suggests that the RTS1 element consists of two elements or functions that can be separated and that only the element that depends on swil and swi3 function is polar. A more detailed study of the cis- and trans-acting factors involved in termination of replication will allow a better understanding of the processes of termination of replication.

\section{Materials and methods}

$2 D$ gels

The 2D gel analysis was performed as described by Brewer and Fangman (1987). Strains were grown in rich YEA media (Moreno et al. 1991), supplemented with $70 \mathrm{mg} / \mathrm{L}$ of uracil, adenine, histidine, and leucine. DNA (50 $\mu \mathrm{g})$ was digested with restriction enzymes and replication intermediates were purified using BND cellulose. The first and second dimensions contained $0.4 \%$ and $1.2 \%$ agarose, respectively. For the direction of replication experiments, electrophoresis in the second dimension were performed with $1.3 \%$ agarose.

\section{Plasmids and S. pombe strains and crosses}

Strains were constructed using standard methods (Moreno et al. 1991) from wild-type strain SP976 (h90, ade6-M210, ura4-D18, leu1-32). Other strains are described in Dalgaard and Klar (1999, 2000). $\Delta$ ARS756 corresponds to a deletion of nucleotides 7829 to 8144 of the mat1P HindIII fragment. Rates of switching were determined from $~ 100$ single-cell pedigrees as described by Miyata and Miyata (1981).

\section{Acknowledgments}

We thank our colleagues at DHHS and MCRI for suggestions and helpful discussions. This research were sponsored by the
National Cancer Institute, U.S. DHHS (AJSK) and the MCRI (JZD). The contents of this article do not necessarily reflect the views or policies of the DHHS, nor does mention of trade names, commercial products, or organizations imply endorsement by the U.S. Government.

The publication costs of this article were defrayed in part by payment of page charges. This article must therefore be hereby marked "advertisement" in accordance with 18 USC section 1734 solely to indicate this fact.

\section{References}

Arcangioli, B. 1998. A site- and strand-specific DNA break confers asymmetric switching potential in fission yeast. $E M B O$ J. 17: 4503-4510.

Arcangioli, B. and Klar, A.J.S. 1991. A novel switch-activating site (SAS1) and its cognate binding factor (SAP1) required for efficient mat1 switching in Schizosaccharomyces pombe. EMBO J. 10: 3025-3032.

Beach, D.H. 1983. Cell type switching by DNA transposition in fission yeast. Nature 305: 682-687.

Beach, D.H. and Klar, A.J.S. 1984. Rearrangements of the transposable mating-type cassettes of fission yeast. Nature 305: 682-687.

Bresch, C., Muller G., and Egel, R. 1968. Genes involved in meiosis and sporulation of a yeast. Mol. Gen. Genet. 102: 301-306.

Brewer, B.J. and Fangman, W.L. 1987. The localization of replication origins on ARS plasmids in $S$. cerevisiae. Cell 51: 463-471.

. 1988. A replication fork barrier at the 3 ' end of yeast ribosomal RNA genes. Cell 55: 637-643.

Dalgaard, J.Z. and Klar, A.J.S. 1999. Orientation of DNA replication establishes mating-type switching pattern in $S$. pombe. Nature 400: 181-184.

- 2000. swi1, and swi3 perform imprinting, pausing and termination of DNA replication in S. pombe. Cell 102: 745751.

. 2001. Does S. pombe exploit the intrinsic asymmetry of DNA synthesis to imprint daughter cells for mating-type switching? Trends Genet. 17: 153-157.

Egel, R. 1989. Mating-type genes, meiosis, and sporulation. In Molecular biology of the fission yeast (ed. A. Nasim, P. Young, and B.F. Johnson), pp. 31-74. Academic Press, San Diego, CA.

Egel, R. and Eie, B. 1987. Cell lineage asymmetry in Schizosaccharomyces pombe: Unilateral transmission of a high-frequency state of mating-type switching in diploid pedigrees. Curr. Genet. 12: 429-433.

Egel, R., Beach, D.H., and Klar, A.J.S. 1984. Genes required for initiation and resolution steps of mating-type switching in fission yeast. Proc. Natl. Acad. Sci. 81: 3481-3485.

Handeli, S., Klar, A., Meuth, M., and Cedar, H. 1989. Mapping replication units in animal cells. Cell 57: 909-920.

Klar, A.J.S. 1987. Differentiated parental DNA strands confer developmental asymmetry on daughter cells in fission yeast. Nature 326: 466-470.

. 1990. The developmental fate of fission yeast cells is determined by the pattern of inheritance of parental and grandparental DNA strands. EMBO J. 9: 1407-1415.

Klar, A.J.S. and Miglio, L.M. 1986. Initiation of meiotic recombination by double-strand DNA breaks in S. pombe. Cell 46: 725-731.

Kobayashi, T., Nomura, M., and Horiuchi, T. 2001. Identification of DNA cis elements essential for expansion of ribo- 
somal DNA repeats in Saccharomyces cerevisiae. Mol. Cell. Biol. 21: 136-147.

Leffak, M. and James, C.D. 1989. Opposite replication polarity of the germ line c-myc gene in HeLa cells compared with that of two Burkitt lymphoma cell lines. Mol. Cell. Biol. 9: 586-593.

Little, R.D., Platt, T.H., and Schildkraut, C.L. 1993. Initiation and termination of DNA replication in human rRNA genes. Mol. Cell. Biol. 13: 6600-6613.

Linskens, M.J. and Huberman, J. 1988. Oganization of replication of ribosomal DNA in Saccharomyces cerevisiae. Mol. Cell. Biol. 8: 4927-4935.

Maundrell, K. 1993. Thiamine-repressible expression vectors pREP and pRIP for fission yeast. Gene 15: 127-130.

Maundrell, K., Hutchison, A., and Shall, S. 1988. Sequence analysis of ARS elements in fission yeast. EMBO J. 7: 22032209.

Miyata, H. and Miyata, M. 1981. Mode of conjugation in homothallic cells of Schizosaccharomyces pombe. I. Gen. Appl. Microbiol. 27: 365-371.

Moreno, S., Klar, A., and Nurse, P. 1991. Molecular genetic analysis of fission yeast Schizosaccharomyces pombe. Methods Enzymol. 194: 795-823.

Santamaria, D., Viguera, E., Martinez-Robles, M.L., Hyrien, O., Hernandez, P., Krimer, D.B., and Schvartzman, J.B. 2000. Bi-directional replication and random termination. Nucleic Acids Res. 28: 2099-2107.

Sanchez, J.A., Kim, S.M., and Huberman, J.A. 1998. Ribosomal DNA replication in the fission yeast, Schizosaccharomyces pombe. Exp. Cell Res. 238: 220-230.

Seidman, M.M., Levine, A.J., and Weintraub, H. 1979. The asymmetric segregation of parental nucleosomes during chromosome replication. Cell 18: 439-449.

Shibahara, K. and Stillman, B. 1999. Replication-dependent marking of DNA by PCNA facilitates CAF-1-coupled inheritance of chromatin. Cell 96: 575-585.

Singh, J. and Klar, A.J.S. 1993. DNA polymerase $\alpha$ is essential for mating-type switching in fission yeast. Nature 361: 271273 .

Styrkarsdottir, U., Egel, R., and Nielsen, O. 1993. The smt-0 mutation which abolishes mating-type switching in fission yeast is a deletion. Curr. Genet. 23: 184-186.

Thon, G. and Klar, A.J.S. 1993. Directionality of fission yeast mating-type interconversion is controlled by the location of the donor loci. Genetics 134: 1045-1054.

Trempe, J.P., Lindstrom, Y.I., and Leffak, M. 1988. Opposite replication polarities of transcribed and nontranscribed histone H5 genes. Mol. Cell. Biol. 8: 1657-1663.

Van't Hof, J. and Lamm, S.S. 1991. Single-stranded replication intermediates of ribosomal DNA replicons of pea. EMBO $I$. 10: 1949-1953.

Wake, R. and King, G. 1997. A tale of two terminators: Crystal structures sharpen the debate on DNA replication fork arrest mechanisms. Structure 5: 1-5.

Wiesendanger, B., Lucchini, R., Koller, T., and Sogo, J.M. 1994. Replication fork barriers in the Xenopus rDNA. Nucleic Acids Res. 22: 5038-5046. 


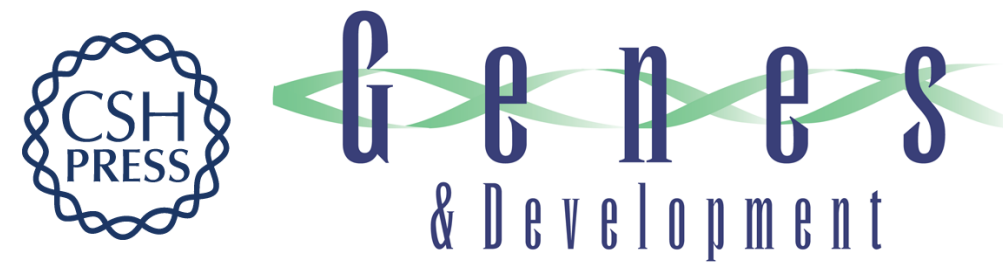

\section{A DNA replication-arrest site $R T S 1$ regulates imprinting by determining the direction of replication at mat1 in S. pombe}

Jacob Z. Dalgaard and Amar J.S. Klar

Genes Dev. 2001, 15:

Access the most recent version at doi:10.1101/gad.200801

References This article cites 34 articles, 8 of which can be accessed free at:

http://genesdev.cshlp.org/content/15/16/2060.full.html\#ref-list-1

License

Email Alerting

Receive free email alerts when new articles cite this article - sign up in the box at the top

Service right corner of the article or click here.

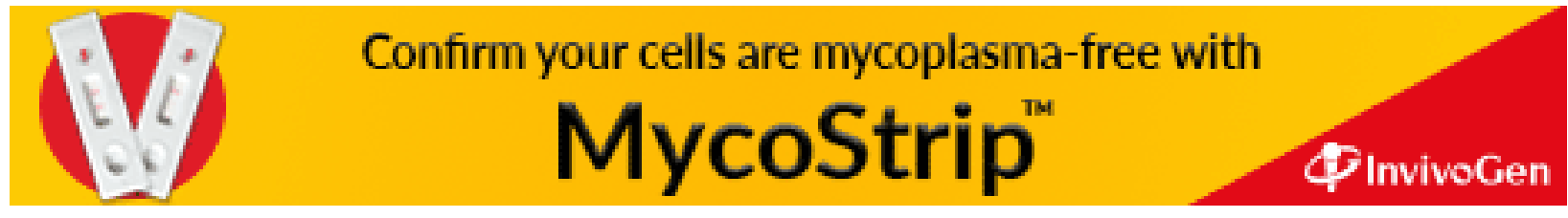

